\title{
PREdiction of ST deviations in lead aVR as a noninvasive tool to predict the infarct-Related coronary artery in patients with acute Inferior-wall Myocardial Infarction (The PREST-RIMI Study)
}

\author{
Santosh Kumar Sinha DM, Vikas Mishra MD, Ramesh Thakur DM, Mukesh Jitendra Jha MD, \\ Amit Goel MD, Ashutosh Kumar MD, Chandra Mohan Varma DM, Vinay Krishna MCh, \\ Avinash Kumar Singh MD, Rupesh Sinha MD
}

SK Sinha, V Mishra, R Thakur, et al. PREdiction of ST deviations in lead $\mathrm{aVR}$ as a noninvasive tool to predict the infarct-Related coronary artery in patients with acute Inferior-wall Myocardial Infarction (The PREST-RIMI Study). Curr Res Cardiol 2016;3(1):17-23.

BACKGROUND: Acute myocardial infarction is one of the most common presentations of coronary artery disease (CAD). Although coronary angiography remains the gold standard for identification of the infarctrelated artery (IRA), conventional 12-lead electrocardiography (ECG) is an essential tool for diagnosis, risk stratification and prognosis. If specific ECG patterns can be recognized, it will be possible to determine the IRA and size of the ventricular area that is jeopardized. The existing ECG algorithms have good sensitivity for the right coronary artery (RCA) and good specificity for the left circumflex artery (LCx) as predictors of IRA in patients with acute inferior-wall myocardial infarction (IWMI), while the specificity for the RCA and sensitivity for the LCx are modest.

OBJECTIVE: To evaluate deviations in lead aVR to predict IRA in patients with IWMI, and to validate several commonly used 12-lead ECG characteristics.

METHODS: A total of 585 consecutive patients with a first occurrence of acute IWMI were analyzed for the association between ECG and IRA diagnosed using coronary angiography. Subsequently, the sensitivity, specificity, positive predictive value, negative predictive value and accuracy of depression in lead aVR, along with various commonly used ECG criteria for predicting the IRA, were estimated using coronary angiographic findings as the gold standard.

RESULTS: The RCA and the LCx were found to be the IRA in 415 (71\%) and 170 (29\%) patients, respectively. It was observed that the absence of ST depression in lead aVR, ST depression in lead I $\geq 0.5 \mathrm{~mm}$, ST depression in lead $\mathrm{aVL}>1 \mathrm{~mm}$ and ST elevation in lead III $>$ ST elevation in lead II had excellent sensitivity and specificity in predicting the RCA as the culprit vessel. Similarly, ST depression in lead aVR $\geq 0.5 \mathrm{~mm}$, the ratio of ST depression in lead V3 to ST elevation in lead III $>1.2$ had excellent sensitivity and specificity in predicting the LCx as the culprit vessel.

CONCLUSION: These findings reveal that evaluation of ST deviations in lead aVR offers good sensitivity and specificity for LCx and RCA occlusions. The authors believe that using multiple ECG criteria may provide earlier and better identification of the IRA.

Key Words: 12-lead electrocardiogram; Coronary angiography; Coronary artery disease; Infarct-related coronary artery; Inferior-wall myocardial infarction; Prognosis

some instances, may prevent particular complications in patients with acute IWMI $(3,4)$.

Many 12-lead ECG-based algorithms have been proposed to predict the IRA in patients with acute MI. These algorithms are simple and user friendly, focusing on one or two leads (3). In acute STEMI patients, the criteria for predicting the culprit artery are usually based on the analysis of ST-segment elevation and ST-segment depression in different leads (6-8). These criteria in isolation have shown good sensitivity for the RCA and good specificity for the LCx in previous studies (9-12). However, the same prediction accuracy may not be reproducible in other populations because of heterogeneity in patients' baseline characteristics and variations in an individual's coronary anatomy. This prompts the need for the development of a more accurate ECG-based algorithm for the prediction of the culprit vessel in inferior-wall STEMI patients. It has been suggested that analysis of multiple ECG leads may minimize errors and considerably improve diagnostic accuracy.

Apart from the commonly used ECG algorithms, ST-segment depression in lead aVR has emerged as a novel predictor of LCx involvement (4,10-13). The current of injury resulting from occlusion in the RCA is, more or less, perpendicular to the axis of lead aVR. On the other hand, the current of injury resulting from occlusion in the RCA or the LCx as culprit artery may facilitate management and, in

LPS Institute of Cardiology, GSVM Medical College, Kanpur, Uttar Pradesh, India

Correspondence: Dr Santosh Kumar Sinha, Department of Cardiology, LPS Institute of Cardiology, GSVM Medical College, Kanpur, Uttar Pradesh India, 208002. Telephone 91-9670220088, fax 91-0512-2556199,2556521, email fionasan@rediffmail.com 
TABLE 1

Baseline characteristics of patients analyzed in the study ( $n=585)$

\begin{tabular}{|c|c|}
\hline Characteristic & \\
\hline \multicolumn{2}{|l|}{ Age, years } \\
\hline Mean \pm SD & $51.2 \pm 16.3$ \\
\hline $20-30$ & $46(08)$ \\
\hline $30-40$ & $109(17)$ \\
\hline $40-50$ & $205(35)$ \\
\hline $50-60$ & $129(22)$ \\
\hline$>60$ & $105(18)$ \\
\hline Sex, male/female, n (\%) & $415(71) / 170(29)$ \\
\hline \multicolumn{2}{|l|}{ Chest pain duration, $\mathrm{h}$} \\
\hline Mean $\pm S D$ & $8.2 \pm 1.31$ \\
\hline$<3$ & $112(19)$ \\
\hline $3-6$ & $199(34)$ \\
\hline $6-12$ & $274(47)$ \\
\hline Heart rate, beats/min, mean \pm SD (range) & $64.0 \pm 18.5(30-112)$ \\
\hline $\mathrm{SBP}, \mathrm{mmHg}$, mean $\pm \mathrm{SD}$ (range) & $101.3 \pm 16.3(74-132)$ \\
\hline $\mathrm{DBP}, \mathrm{mmHg}$, mean $\pm \mathrm{SD}$ (range) & $72.4 \pm 8.6(56-94)$ \\
\hline Cigarette smoker & $126(21.5)$ \\
\hline Diabetes & $104(17.7)$ \\
\hline Hypertension & $113(19.3)$ \\
\hline Dyslipidemia & $128(21.8)$ \\
\hline Obesity & $137(23.4)$ \\
\hline Family history of premature coronary artery disease & $36(6.1)$ \\
\hline
\end{tabular}

Data presented as $n$ (\%) unless otherwise indicated. DBP Diastolic blood pressure; SBP Systolic blood pressure

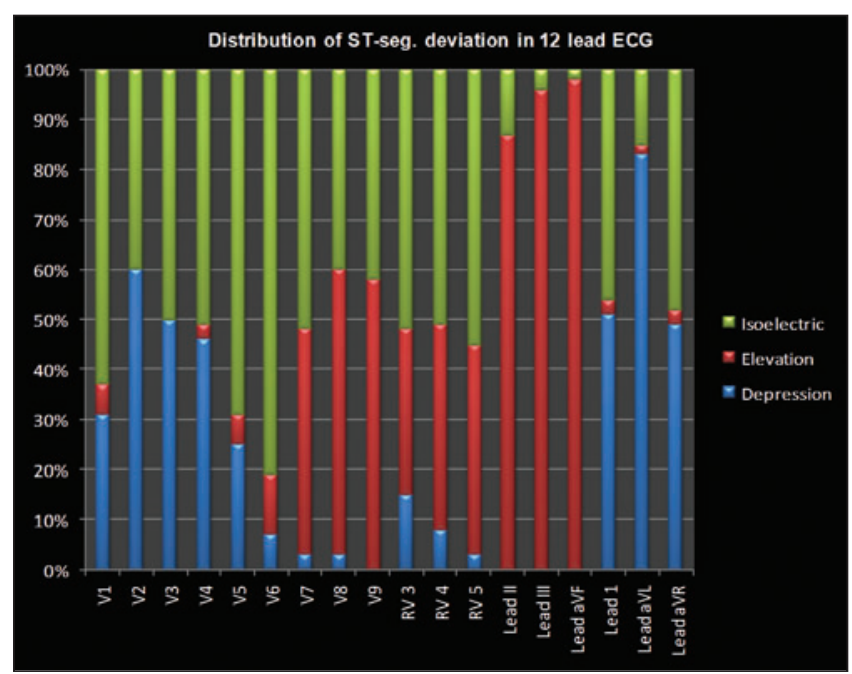

Figure 1) 12-lead electrocardiogram analysis depicting the distribution of ST-segment deviation in the study patients

LCx has a mean vector that forms an obtuse angle with the axis of aVR. Therefore, significant ST-segment depression in lead aVR is more likely to occur with LCx occlusion $(10,13)$. Despite these observations, ST-segment deviation in lead aVR is usually ignored during the ECG analysis (4). Accordingly, we conducted the present study with the aim of analyzing depression in lead aVR as a newer criterion to identify the IRA in patients with inferior-wall STEMI. Subsequently, we attempted to develop a new and more accurate ECG algorithm by incorporating ST-segment deviation in other leads, along with the reciprocal changes in lead aVR and also to validate several commonly used 12-lead ECG algorithms in predicting the IRA.
TABLE 2

Distribution of ST-segment deviation in the study patients based on 12-lead electrocardiogram analysis

\begin{tabular}{lccc}
\hline \multirow{3}{*}{ Lead } & \multicolumn{3}{c}{ ST-segment deviation } \\
\cline { 2 - 4 } VI & Depression & Elevation & Isoelectric \\
V2 & $181(31.0)$ & $35(6.0)$ & $369(63.0)$ \\
V3 & $291(60.0)$ & $0(0.0)$ & $234(40.0)$ \\
V4 & $269(46.0)$ & $0(0.0)$ & $293(50.0)$ \\
V5 & $14(25.0)$ & $35(6.0)$ & $299(51.0)$ \\
V6 & $41(7.0)$ & $69(12.0)$ & $404(69.0)$ \\
V7 & $17(3.0)$ & $234(45.0)$ & $374(81.0)$ \\
V8 & $17(3.0)$ & $333(57.0)$ & $235(40.0)$ \\
V9 & $0(0.0)$ & $340(58.0)$ & $245(42.0)$ \\
RV3 & $85(15.0)$ & $195(33.0)$ & $305(52.0)$ \\
RV4 & $47(8.0)$ & $239(41.0)$ & $299(51.0)$ \\
RV5 & $17(3.0)$ & $245(42.0)$ & $323(55.0)$ \\
II & $0(0.0)$ & $508(87.0)$ & $79(13.0)$ \\
III & $0(0.0)$ & $562(96.0)$ & $23(4.0)$ \\
aVF & $0(0.0)$ & $573(98.0)$ & $12(2.0)$ \\
1 & $299(51.0)$ & $17(3.0)$ & $269(46.0)$ \\
aVL & $486(83.0)$ & $12(2.0)$ & $87(15.0)$ \\
aVR & $287(49.0)$ & $17(3.0)$ & $281(48.0)$ \\
\hline Data pres) & & \\
\hline
\end{tabular}

Data presented as $n$ (\%)

\section{METHODS}

The present study was a prospective, single-centre trial conducted between January and July 2015 in the Department of Cardiology, LPS Institute of Cardiology, GSVM Medical College, Kanpur and UP, India. Five hundred eighty-five consecutive patients presented with a first occurrence of acute inferior-wall STEMI and were enrolled in the study. The enrollment criteria were: patients diagnosed with acute inferior-wall STEMI based on 12-lead ECG, as evidenced by $\geq 1 \mathrm{~mm}$ ST elevation in at least two of the inferior leads II, III and aVF; and patients who subsequently underwent coronary angiography and percutaneous coronary intervention (PCI), either as primary $\mathrm{PCI}$ or pharmacoinvasive PCI. The exclusion criteria were: patients with a history of STEMI, coronary artery bypass surgery or PCI; ECG suggesting bundle branch block or left ventricular hypertrophy; finding of normal epicardial coronaries on angiography; no consensus on culprit coronary artery after angiography; electrolyte abnormality; and certain conditions that can influence ST segment on ECG (eg, suspected myocarditis, pericarditis, hypothermia, receiving amiodarone treatment, etc). Informed consent was obtained from each patient enrolled in the study. The study protocol was approved by the institutional ethics committee and performed in accordance with the Declaration of Helsinki.

\section{Data collection}

Enrolled patients underwent ECG, cardiac enzyme evaluation, routine biochemistry, two-dimensional echocardiography and coronary angiography as a part of their diagnostic procedure to identify the involved coronary artery occlusion in acute STEMI. The 12-lead ECG was recorded for all patients at a speed of $25 \mathrm{~mm} / \mathrm{s}$ and voltage of $10 \mathrm{~mm} / \mathrm{mV}$ on a 12-channel device (Mortara ELI 250 ECG, Burdick Inc, USA). ST-segment deviation was measured at $60 \mathrm{~ms}$ from the J point in all leads. Measurements were recorded to the nearest $0.5 \mathrm{~mm}(0.05 \mathrm{mV})$. The preceding TP segment was used as the isoelectric reference line. The mean of three beats was considered to be representative. Angiography was performed via either the radial or femoral route after Allen's test (5 Fr [TIG Cath-Teumo Inc, USA]; 5 Fr JL/JR [Medtronic, USA]). Subsequently, the culprit artery was identified based on the angiographic characteristics of the occlusion (ie, occlusion due to thrombus/plaque formation, dissection or ulceration with decreased contrast density). Coronary artery stenosis $>70 \%$ was considered to be 


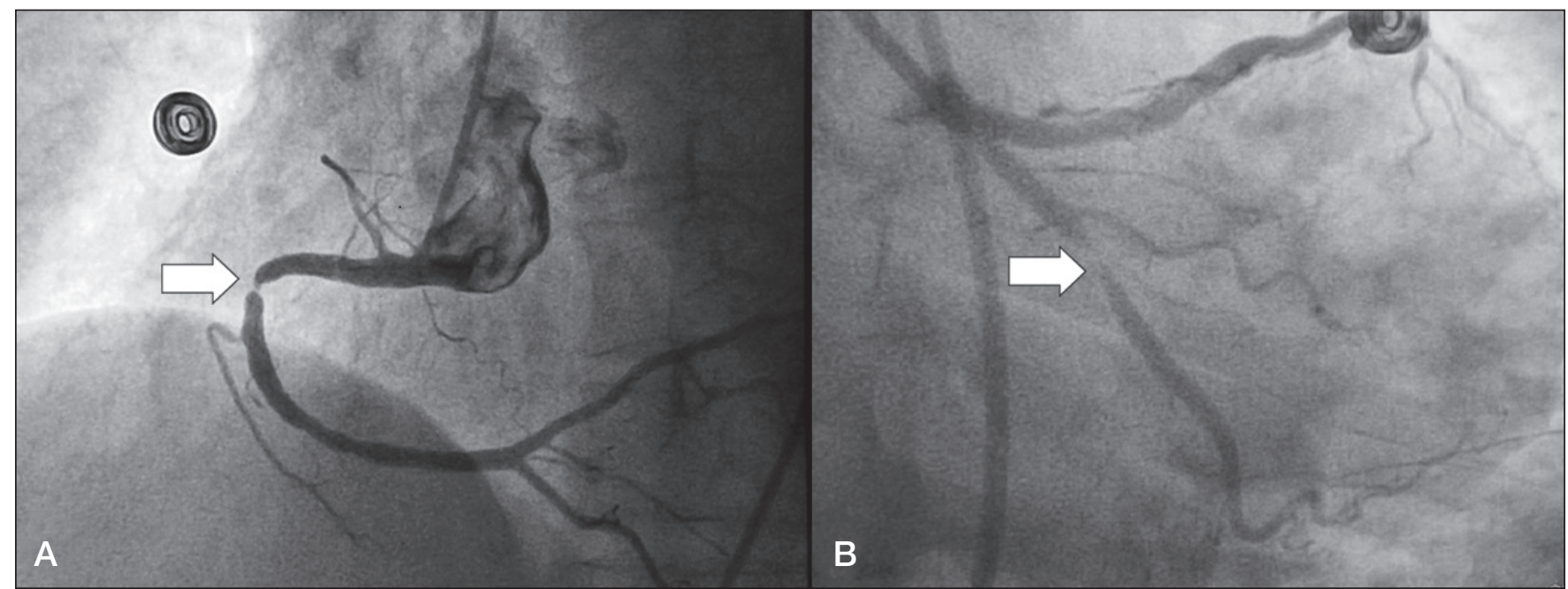

Figure 2) Coronary angiography. Right coronary artery (A) and left circumflex artery (B) occlusions (arrows) identified as the infarct-related artery in patients with inferior-wall myocardial infarction

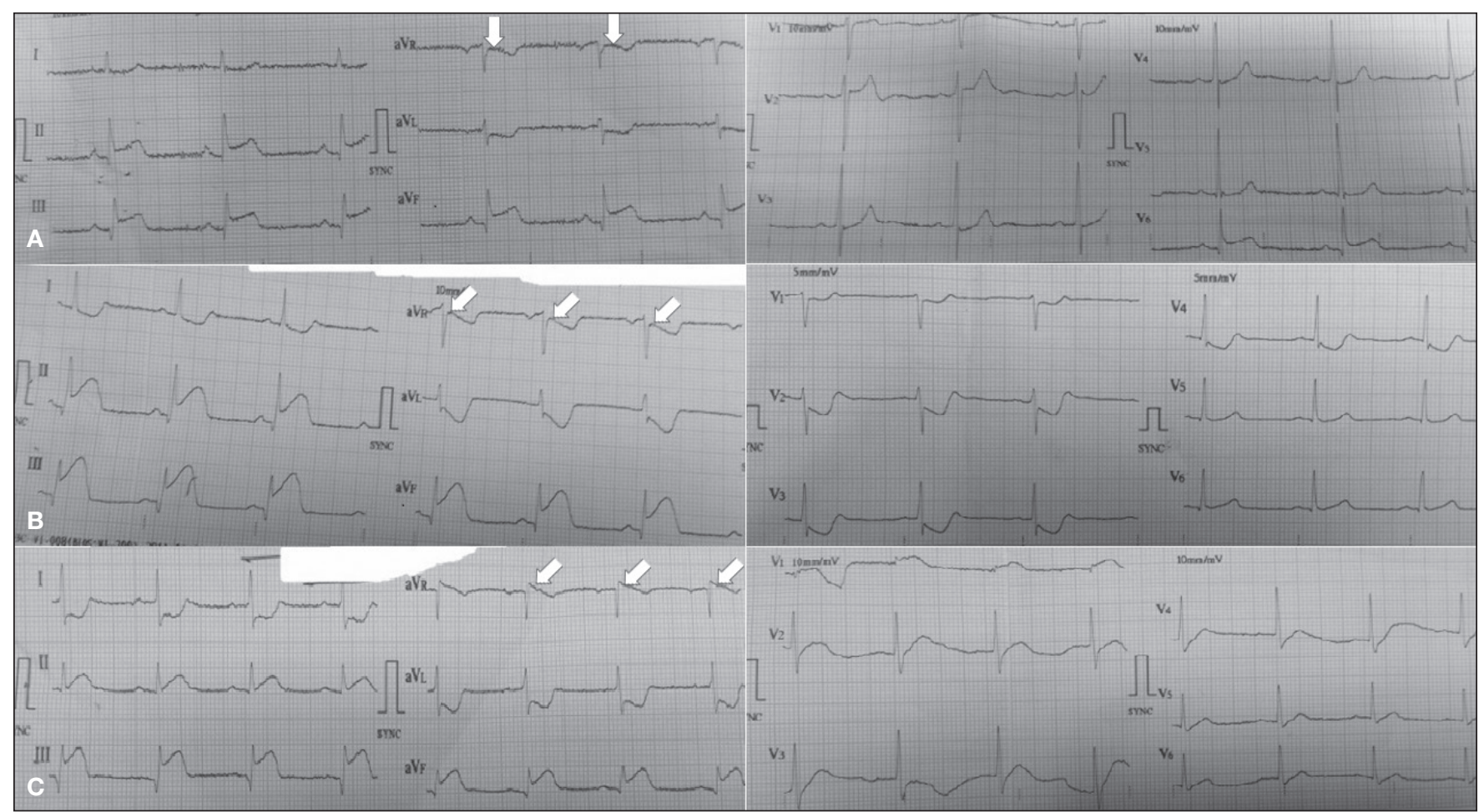

Figure 3) Electrocardiographic characteristics of right coronary artery (A) and left circumflex artery (B,C) occlusions (arrows) indentifying the infarct-related artery in patients with inferior-wall myocardial infarction

an obstructive lesion. Patients were divided into RCA and LCx groups based on the angiographic characteristics suggesting obstructive lesions in the RCA and the LCx vessels, respectively.

\section{Statistical analysis}

Continuous variables are expressed as mean $\pm \mathrm{SD}$, while discrete variables are presented as frequency and percentage. The $\chi^{2}$ test was used to analyze the association between ECG and coronary angiography findings for identifying the IRA. In addition, sensitivity, specificity, positive predictive value, negative predictive value and accuracy of ST-segment deviation in lead aVR, along with various commonly used ECG criteria for predicting the culprit artery, were estimated using coronary angiographic findings as the gold standard. All analyses were performed using STATA version 8.0 (StataCorp, USA) and $\mathrm{P}<0.05$ was considered to be statistically significant.

\section{RESULTS}

Baseline characteristics

The baseline characteristics of the enrolled patients are summarized in Table 1 . The mean $( \pm \mathrm{SD})$ age of the patients was $51.2 \pm 16.3$ years. Age stratification revealed that more than one-third (35\%) of patients were between 40 and 50 years of age, followed by 51 to 60 years (22\%), 31 to 40 years $(17 \%), 21$ to 30 years $(8 \%)$ and $>60$ years $(18 \%)$. Furthermore, the majority $(71 \%)$ of enrolled patients were male. The mean duration of pain was $8.2 \pm 1.31 \mathrm{~h}$. In particular, the duration of pain was $6 \mathrm{~h}$ to $12 \mathrm{~h}$ in $47 \%$ of patients, $3 \mathrm{~h}$ to $6 \mathrm{~h}$ in $34 \%$ and $<3 \mathrm{~h}$ in $19 \%$. Figure 1 illustrates the distribution of ST-segment deviation based on 12-lead ECG data. Briefly, the ST-segment depression in lead V1, lead V2, lead I and lead aVL were reported in 181 (31\%), 351 (60\%), 299 (51\%) and $486(83 \%)$ patients, respectively (Table 2). Angiographic findings revealed that the RCA was the culprit coronary vessel in 


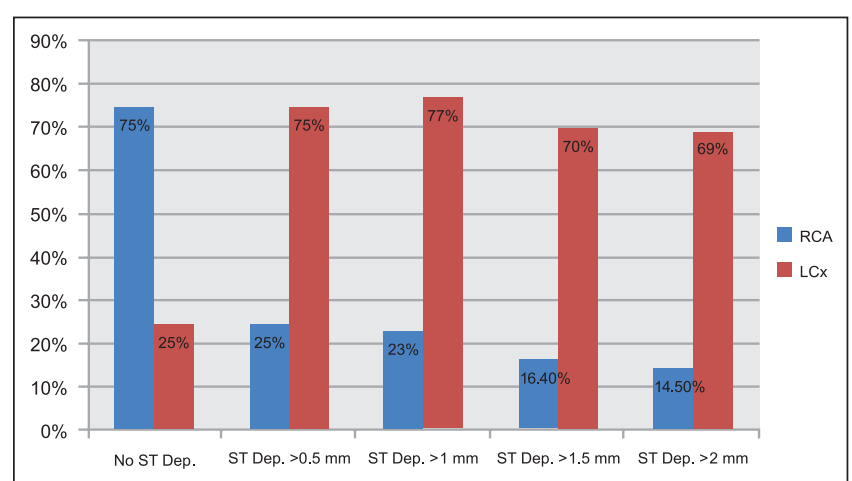

Figure 4) Comparison of accuracy of lead aVR as a predictor of the infarctrelated artery. Dep Depression; LCx Left circumflex artery; RCA Right coronary artery

$415(71 \%)$ patients, while the LCx was the culprit coronary vessel in the remaining 170 (29\%) (Figure 2). Figure 3 presents ECG data, with the RCA (Figure 3A) and the LCx (Figures 3B and 3C) as the IRA in patients with IWMI after coronary angiography.

ST-deviation in lead aVR as a predictor of the IRA

ST depression in lead aVR $\geq 0.5 \mathrm{~mm}$ was observed in 130 (31.5\%) patients in the RCA group and $150(88.5 \%)$ in the LCx group; the difference was statistically significant $(\mathrm{P}<0.001)$. Furthermore, 85 (20.7\%) patients in the RCA group and 50 (29.9\%) in the LCx group exhibited ST depression in lead aVR $\geq 1 \mathrm{~mm}$; however, the difference was not statistically significant (Table 3 ). Similarly, there was no significant difference between the RCA and LCx groups in the proportion of patients with ST depression in lead aVR $\geq 2 \mathrm{~mm}$ (Table 3 ). Lead aVR with no ST-segment depression exhibited good specificity (90\%) and fair sensitivity (68.6\%), with good accuracy (75\%) in predicting RCA as the culprit vessel (Table 4). However, ST depression in lead aVR $\geq 0.5 \mathrm{~mm}, \geq 1 \mathrm{~mm}, \geq 1.5 \mathrm{~mm}$, and $\geq 2 \mathrm{~mm}$ displayed poor sensitivity and specificity in predicting RCA as the culprit vessel (Table 4). On the other hand, only ST depression in lead aVR $\geq 0.5 \mathrm{~mm}$ and $\geq 1 \mathrm{~mm}$ had good sensitivity in predicting the LCx as the culprit vessel, while ST depression in lead aVR $\geq 2 \mathrm{~mm}$ had good specificity (91.4\%) in predicting the LCx as the culprit vessel (Table 5). When ST depression was analyzed as no ST depression or depression $\geq 0.5 \mathrm{~mm}, \geq 1 \mathrm{~mm}, \geq 1.5 \mathrm{~mm}$ and $\geq 2 \mathrm{~mm}$ in lead aVR to accurately predict the IRA, differences were statistically significant (Figure 4).

Ratio of ST-depression in lead V3 to ST elevation in lead III as a predictor of the culprit vessel

A joint observation of ST-depression in lead V3 and ST-elevation in lead III was reported in 157 (38.0\%) patients from the RCA group and $80(47.1 \%)$ in the LCx group (Table 3$)$. Of these, $92(22.2 \%)$ patients in the RCA group and $82(48.8 \%)$ in the LCx group exhibited the ratio of ST depression in lead V3 to ST elevation in lead III >1.2; the difference was statistically significant $(\mathrm{P}=0.02)$ (Table 3$)$. Further, the ratio of ST depression in lead V3 to ST elevation in lead III $>1.2$ and the ratio of ST depression in lead V3 to ST elevation in lead III $\leq 1.2$ had fair sensitivity and specificity in predicting LCX and RCA as culprit vessels respectively (Tables 4 and 5).

ST-elevation in lead II and lead III as predictors of culprit vessel ST elevation in lead II was present in $342(82.6 \%)$ patients in the RCA group and 156 (92\%) in the LCx group; the difference was not significant $(\mathrm{P}>0.05)$ (Table 3$)$. Conversely, ST elevation in lead III was present in all $415(100 \%)$ patients in the RCA group and 156 $(91.7 \%)$ in the LCx group, with significant difference $(\mathrm{P}=0.007)$ (Table 3). Notably, 359 (86.6\%) patients from the RCA group and 38 (22.4\%) from the LCx group had ST elevation in lead III > ST elevation in lead II (Table 3). It was also observed that ST elevation in lead III $>$ ST elevation in lead II and ST elevation in lead II $>$ ST elevation

\section{TABLE 3}

Analysis of association between elctrocardiographic characteristics and the culprit coronary artery

\begin{tabular}{|c|c|c|c|}
\hline ST-segment deviation & $\mathrm{RCA}(\mathrm{n}=415)$ & LCx $(n=170)$ & $\mathbf{P}^{*}$ \\
\hline No ST depression in lead aVR & $280(67.5)$ & $19(11.5)$ & $<0.001^{\dagger}$ \\
\hline $\begin{array}{l}\text { ST depression in lead aVR } \\
\geq 0.5 \mathrm{~mm}\end{array}$ & $130(31.5)$ & $150(88.5)$ & $<0.001^{\dagger}$ \\
\hline $\begin{array}{l}\text { ST depression in lead aVR } \\
\geq 1 \mathrm{~mm}\end{array}$ & $85(20.7)$ & $50(29.9)$ & 0.28 \\
\hline $\begin{array}{l}\text { ST depression in lead aVR } \\
\geq 1.5 \mathrm{~mm}\end{array}$ & $46(11.3)$ & $19(11.5)$ & 0.69 \\
\hline $\begin{array}{l}\text { ST depression in lead aVR } \\
\geq 2 \mathrm{~mm}\end{array}$ & $35(8.5)$ & $14(8.1)$ & 0.52 \\
\hline $\begin{array}{l}\text { ST-segment depression in lead } \\
\text { V3 and ST elevation in lead III }\end{array}$ & $157(38.0)$ & $80(47.1)$ & 0.15 \\
\hline $\begin{array}{l}\text { Ratio of ST depression in lead V3 } \\
\text { to ST elevation in lead III >1.2 }\end{array}$ & $92(22.2)$ & $82(48.8)$ & $0.003^{\dagger}$ \\
\hline $\begin{array}{c}\text { Ratio of ST depression in lead V3 } \\
\text { to ST elevation in lead III } \leq 1.2\end{array}$ & $322(77.8)$ & $87(51.2)$ & \\
\hline ST elevation in lead II & $342(82.6)$ & $156(92.0)$ & 0.08 \\
\hline ST elevation in lead III & $415(100.0)$ & $158(93.1)$ & $0.007^{\dagger}$ \\
\hline ST elevation in lead II and lead III & $306(73.7)$ & $148(87.4)$ & $0.01^{\dagger}$ \\
\hline $\begin{array}{l}\text { ST elevation in lead III > } \\
\text { ST elevation in lead II }\end{array}$ & $359(86.6)$ & $38(22.4)$ & $<0.001^{\dagger}$ \\
\hline $\begin{array}{l}\text { ST elevation in lead II > } \\
\text { ST elevation in lead III }\end{array}$ & $55(13.4)$ & 131(77.6) & \\
\hline ST depression in lead I $\geq 0.5 \mathrm{~mm}$ & $284(68.6)$ & $29(17.2)$ & $<0.001^{*}$ \\
\hline ST depression in lead I $\geq 1 \mathrm{~mm}$ & $132(31.9)$ & $13(8.1)$ & $0.006^{\dagger}$ \\
\hline ST depression lead $\mathrm{aVL} \geq 0.5 \mathrm{~mm}$ & $380(91.7)$ & $74(43.7)$ & $<0.001^{\dagger}$ \\
\hline ST depression lead aVL $\geq 1 \mathrm{~mm}$ & $302(73.3)$ & $47(27.6)$ & $<0.001^{\dagger}$ \\
\hline $\begin{array}{l}\text { ST depression in lead aVR > } \\
\text { ST depression in lead aVL }\end{array}$ & $14(3.3)$ & $29(17.2)$ & $0.01^{\dagger}$ \\
\hline $\begin{array}{l}\text { ST depression in lead aVL > } \\
\text { ST depression in lead aVR }\end{array}$ & $117(28.2)$ & $29(17.2)$ & 0.20 \\
\hline
\end{tabular}

Data presented as $n$ (\%) unless otherwise indicated. ${ }^{*}$ Chi-squared test; 'Statistically significant; LCX Left circumflex artery; RCA Right coronary artery

in lead III had good sensitivity and specificity in predicting RCA and LCx as culprit vessels, respectively (Tables 4 and 5).

ST depression in lead I as a predictor of the culprit vessel Of patients with RCA as the IRA, the ST-segment depression in lead $\mathrm{I} \geq 0.5 \mathrm{~mm}$ and $>1 \mathrm{~mm}$ were observed in $284(68.6 \%)$ and $131(31.9 \%)$ patients, respectively (Table 2$)$. On the other hand, ST depression in lead $\mathrm{I} \geq 0.5 \mathrm{~mm}$ and $>1 \mathrm{~mm}$ was observed in $29(17.2 \%)$ and $13(8.1 \%)$ patients from the LCx group, respectively; the difference was statistically significant $(\mathrm{P}<0.01)$ (Table 3$)$. Furthermore, the ST depression in lead I $\geq 0.5$ had good specificity $(93.3 \%)$ and fair sensitivity $(55.7 \%)$ in predicting the RCA as the culprit vessel (Table 4) and poor sensitivity and specificity in predicting the LCx as the culprit vessel (Table 5).

ST-deviation in lead aVL as a predictor of the culprit vessel ST depression in lead aVL $\geq 0.5 \mathrm{~mm}$ and $>1 \mathrm{~mm}$ was observed in $380(91.7 \%)$ and $302(73.3 \%)$ patients in the RCA group, respectively (Table 3). Similarly, ST depression in lead aVL $\geq 0.5 \mathrm{~mm}$ and $>1 \mathrm{~mm}$ was observed in $74(43.7 \%)$ and $47(27.6 \%)$ patients in the LCx group, respectively; the difference was statistically significant $(\mathrm{P}<0.01)$ (Table 3). Furthermore, the ST depression in lead aVL $\geq 0.5 \mathrm{~mm}$ had good sensitivity $(91.4 \%)$, fair specificity $(56.7 \%)$ and good accuracy $(81.0 \%)$ in predicting RCA as the culprit vessel (Table 3$)$. The ST depression in lead aVL $>1 \mathrm{~mm}$ also exhibited reasonable sensitivity $(72.9 \%)$ and specificity $(73.3 \%)$ in predicting RCA as the culprit vessel (Table 4). Our observations also revealed that ST depression in lead $\mathrm{aVL} \geq 0.5 \mathrm{~mm}$ and $>1 \mathrm{~mm}$ had poor sensitivity and specificity in predicting the LCx as the culprit vessel (Table 5). 
TABLE 4

Sensitivity and specificity of electrocardiographic parameters in predicting the right coronary artery as the culprit vessel

\begin{tabular}{|c|c|c|c|c|c|}
\hline \multirow[b]{2}{*}{ ST-segment deviation } & \multirow[b]{2}{*}{ Sensitivity } & \multirow[b]{2}{*}{ Specificity } & \multicolumn{2}{|c|}{ Predictive value } & \multirow[b]{2}{*}{ Accuracy } \\
\hline & & & Positive & Negative & \\
\hline No ST depression in lead aVR & 68.6 & 90.0 & 94.1 & 55.1 & 75.0 \\
\hline ST depression in lead aVR $\geq 0.5 \mathrm{~mm}$ & 31.4 & 10.0 & 44.9 & 5.9 & 25.0 \\
\hline ST depression in lead aVR $\geq 1 \mathrm{~mm}$ & 21.4 & 26.7 & 40.9 & 12.7 & 23.0 \\
\hline ST depression in lead aVR $\geq 1.5 \mathrm{~mm}$ & 14.3 & 27.3 & 50.0 & 5.9 & 16.4 \\
\hline ST depression in lead aVR $\geq 2 \mathrm{~mm}$ & 11.1 & 37.5 & 54.5 & 5.9 & 14.5 \\
\hline Ratio of ST depression in lead V3 to ST elevation in lead III $\leq 1.2$ & 20.0 & 52.4 & 44.4 & 25.6 & 31.1 \\
\hline ST elevation in lead III > ST elevation in lead II & 84.7 & 77.8 & 89.3 & 70.0 & 82.5 \\
\hline No ST depression lead I & 21.4 & 92.9 & 75.0 & 54.2 & 57.1 \\
\hline ST depression in lead I $\geq 0.5 \mathrm{~mm}$ & 55.7 & 93.3 & 95.1 & 47.5 & 67.0 \\
\hline ST depression in lead I $\geq 1 \mathrm{~mm}$ & 32.9 & 93.3 & 92.0 & 37.3 & 51.0 \\
\hline ST depression lead $\mathrm{aVL} \geq 0.5 \mathrm{~mm}$ & 91.4 & 56.7 & 83.1 & 73.9 & 81.0 \\
\hline ST depression lead $\mathrm{aVL} \geq 1 \mathrm{~mm}$ & 72.9 & 73.3 & 86.4 & 53.7 & 73.0 \\
\hline ST depression in lead aVL > ST depression in lead aVR & 28.6 & 83.3 & 80.0 & 33.3 & 45.0 \\
\hline
\end{tabular}

Data presented as \%

TABLE 5

Sensitivity and specificity of electrocardiographic parameters in predicting the left circumflex artery as the culprit vessel

\begin{tabular}{|c|c|c|c|c|c|}
\hline \multirow[b]{2}{*}{ ST-segment deviation } & \multirow[b]{2}{*}{ Sensitivity } & \multirow[b]{2}{*}{ Specificity } & \multicolumn{2}{|c|}{ Predictive value } & \multirow[b]{2}{*}{ Accuracy } \\
\hline & & & Positive & Negative & \\
\hline No ST depression in lead aVR & 10.0 & 31.4 & 5.9 & 44.9 & 25.0 \\
\hline ST depression in lead aVR $\geq 0.5 \mathrm{~mm}$ & 90.0 & 68.6 & 55.1 & 94.1 & 75.0 \\
\hline ST depression in lead aVR $\geq 1 \mathrm{~mm}$ & 73.3 & 78.6 & 59.5 & 87.3 & 77.0 \\
\hline ST depression in lead aVR $\geq 1.5 \mathrm{~mm}$ & 26.7 & 88.6 & 50.0 & 73.8 & 70.0 \\
\hline ST depression in lead aVR $\geq 2 \mathrm{~mm}$ & 16.7 & 91.4 & 45.5 & 71.9 & 69.0 \\
\hline Ratio of ST depression in lead V3 to ST elevation in lead III >1.2 & 47.6 & 78.0 & 52.6 & 74.4 & 34.4 \\
\hline ST elevation in lead II > ST elevation in lead III & 77.8 & 84.7 & 70.0 & 89.3 & 82.5 \\
\hline No ST depression lead I & 7.1 & 78.6 & 25.0 & 45.8 & 42.8 \\
\hline ST depression in lead I $\geq 0.5 \mathrm{~mm}$ & 6.7 & 44.3 & 4.9 & 52.5 & 33.0 \\
\hline ST depression in lead $\mathrm{I} \geq 1 \mathrm{~mm}$ & 6.7 & 67.1 & 8.0 & 62.7 & 49.0 \\
\hline ST depression lead aVL $\geq 0.5 \mathrm{~mm}$ & 43.3 & 8.6 & 16.9 & 26.1 & 19.0 \\
\hline ST depression lead aVL $\geq 1 \mathrm{~mm}$ & 26.7 & 27.1 & 13.6 & 46.3 & 27.0 \\
\hline ST depression in lead aVR $>$ ST depression in lead $\mathrm{aVL}$ & 16.7 & 97.1 & 71.4 & 73.1 & 73.0 \\
\hline
\end{tabular}

Data presented as \%

TABLE 6

Study comparisons of parameters for predicting the right coronary and left circumflex arteries as the culprit vessel

\begin{tabular}{|c|c|c|c|c|c|}
\hline \multirow[b]{2}{*}{ Criteria } & \multirow[b]{2}{*}{ Study } & \multicolumn{4}{|c|}{ Right coronary artery occlusion } \\
\hline & & Sensitivity & Specificity & PPV & NPV \\
\hline \multirow[t]{4}{*}{ ST elevation in III > ST elevation in lead II predicts } & Present study & 84.7 & 77.8 & 89.3 & 70.0 \\
\hline & Elhammady et al (10) & 92.0 & 78.0 & - & - \\
\hline & Kanei et al (11) & 94.0 & 58.0 & 91.0 & 69.0 \\
\hline & Nair and Glancy (12) & 96.0 & 40.0 & 89.0 & 67.0 \\
\hline \multirow[t]{2}{*}{ Ratio of ST depression in lead V3 to ST elevation in lead III $\leq 1.2$} & Our study & 20.0 & 52.4 & 44.4 & 25.6 \\
\hline & ASSENT $4 \mathrm{PCl}(9)$ & 91.0 & 32.0 & 79.0 & 55.0 \\
\hline \multirow[t]{3}{*}{ ST depression in lead I } & Present study ( $\geq 0.5 \mathrm{~mm})$ & 55.7 & 93.3 & 95.1 & 47.5 \\
\hline & Kanei et al (11) & 86.0 & 63.0 & 91.0 & 50.0 \\
\hline & ASSENT $4 \mathrm{PCl}(9)$ & 84.0 & 60.0 & 87.0 & 53.0 \\
\hline \multirow[t]{2}{*}{ ST depression in lead aVL > ST depression in lead aVR } & Present study & 28.6 & 83.3 & 80.0 & 33.3 \\
\hline & & \multicolumn{4}{|c|}{ Left circumflex artery occlusion } \\
\hline \multirow[t]{4}{*}{ ST depression in lead aVR $\geq 1 \mathrm{~mm}$} & Present study & 73.3 & 78.6 & 59.5 & 87.3 \\
\hline & Elhammady et al (10) & 86.0 & 92.0 & - & - \\
\hline & Knei et al (11) & 54.0 & 86.0 & 45.0 & 91.0 \\
\hline & Nair and Glancy (12) & 80.0 & 96.0 & 80.0 & 96.0 \\
\hline \multirow[t]{3}{*}{ ST elevation in lead II > ST elevation in lead III } & Present study & 77.8 & 84.7 & 70.0 & 89.3 \\
\hline & Elhammady et al (10) & 80.0 & 93.0 & - & - \\
\hline & Nair and Glancy(12) & 40.0 & 96.0 & 67.0 & 89.0 \\
\hline \multirow[t]{2}{*}{ Ratio of ST depression in lead V3 to ST elevation in lead III >1.2 } & Present study & 47.6 & 78.0 & 52.6 & 74.4 \\
\hline & Kanei et al (11) & 21.0 & 98.0 & 67.0 & 85.0 \\
\hline ST depression in lead aVR > ST depression in lead aVL & Present study & 16.7 & 97.1 & 71.4 & 73.1 \\
\hline
\end{tabular}

Data presented as \%. ASSENT 4 PCI The Assessment of the Safety and Efficacy of a New Treatment Strategy for Acute Myocardial Infarction trial; NPV Negative predictive value; PPV Positive predictive value 
ST-deviation in lead aVL and lead aVR as a predictor of the culprit vessel

ST depression in lead aVR was greater than ST depression in lead aVL in $14(3.3 \%)$ and $29(17.2 \%)$ patients in the RCA and LCx groups, respectively; with statistically significant difference $(\mathrm{P}=0.01)$ (Table 3). ST depression in lead aVL > ST depression in lead aVR exhibited fair sensitivity (28.6\%) and good specificity (83.3\%) in predicting RCA as the culprit vessel (Table 4). Similarly, ST depression in lead aVR > ST depression in lead aVL exhibited poor sensitivity (16.7\%) and good specificity $(97.1 \%)$ with good accuracy $(73 \%)$ in predicting the LCx as the culprit vessel (Table 5).

\section{DISCUSSION}

The 12-lead ECG has been the most feasible, useful, inexpensive and universally available tool for the initial evaluation, risk stratification, triage and guidance of therapy in patients with a suspected acute ischemic event (4). Additionally, ECG helps to predict the culprit artery and locate the lesion within the IRA, thus providing clinically important information to augment clinical decision making and tailor reperfusion therapy (4). The importance of ECG changes and their relationship with the IRA was recognized in the 1980s (9). Since then, numerous studies have described various ECG criteria to identify the IRA in patients with inferior-wall STEMI (7-12). For example, ST-segment depression in lead I $\geq 0.5 \mathrm{~mm}$; ratio of ST-segment elevation in II/III $<1$; ratio of ST-segment depression in $\mathrm{V} 3$ to ST-segment elevation in III $\leq 1.2$; and ST-segment depression in aVL $>1$ predicts RCA occlusion. In contrast, ST-segment isoelectric or elevation in lead I; ratio of ST-segment elevation in lead II to ST-segment elevation in lead III $>1$; and ratio of ST-segment depression in V3 to ST-segment elevation in III $>1.2$ predicts LCx occlusion. Based on these findings, Fiol et al (6) developed an algorithm that was able to predict proximal RCA, distal RCA and LCx occlusion with high accuracy.

It should be noted that lead aVR has traditionally received less attention in clinical evaluation among all ECG leads, probably because it is directionally nonadjacent to any other ECG lead (4). We believe that this 'relative isolation', however, is also the very reason why changes in lead aVR could be particularly important. Hence, the present study was designed to validate the exiting ECG criteria and search for newer aVR criteria to predict the culprit coronary artery in patients with acute IWMI. In the present study, the LCx was the culprit artery in patients with ST depression in lead aVR more than those without ST-segment depression. These results were consistent with previously reported studies $(4,11,13)$. Accordingly, Radhakrishnan et al (4) showed that the amount of ST-segment depression in lead aVR is an accurate predictor of IRA in pateints with IWMI.

In our study, we observed that the commonly used ECG criteria had variable sensitivity and specificity to differentiate between LCx and RCA lesions in patients with acute IWMI. A brief review of previous studies $(3,13)$ in a similar context (Table 6) suggests that ST elevation in lead III > ST elevation in lead II - an ECG criterion - has highest sensitivity (96\% [12]) and specificity (78\% [4]) for predicting $\mathrm{RCA}$ as the culprit vessel. Conversely, ST depression $>1 \mathrm{~mm}$ in lead aVR exhibits highest sensitivity $(86 \%[10])$ in predicting the LCx as the culprit vessel. Furthermore, the ratio of ST depression in lead V3 to ST elevation in lead III $>1.2$ had the highest specificity (11) in predicting the LCx as the culprit vessel (98\%). Overall, the sensitivity of existing ECG criteria ranges from $84 \%$ to $96 \%$ for RCA and $21 \%$ to $86 \%$ for the LCx. Similarly, the specificity of the existing ECG criteria ranges from $40 \%$ to $78 \%$ for RCA and $72 \%$ to $98 \%$ for the LCx (3-13). These data indicate that the existing criteria already have good sensitivity for RCA and good specificity for LCx localization, while the specificity for RCA and sensitivity for LCx ranges from poor to fair. In the present study, we used ST-depression in lead aVR as a novel criterion to predict the culprit vessel. Through this, we found both good sensitivity and good specificity for LCx and good specificity for the RCA. For LCx localization, ST depression $\geq 0.5 \mathrm{~mm}$ in lead aVR exhibited the highest sensitivity (90\%), while ST depression $\geq 0.5$ $\mathrm{mm}$ in lead aVL displayed good specificity (91.4\%). In addition, novel parameter of ST depression in lead aVR > lead aVL exhibited very good specificity $(97.1 \%)$ for LCx occlusion. For RCA localization, no ST depression in lead aVR exhibited good specificity (90\%), while ST depression in lead aVL > lead aVR displayed good specificity (83.3\%).

The anatomy of the coronary arteries plays an important role in exhibiting these ECG characteristics among patients with acute IWMI; hence, its understanding is vital. Briefly, the RCA usually terminates at the posterior descending artery and a few posterolateral branches to supply blood to the inferior myocardium and inferior part of the inferoposterior wall, respectively. On the other hand, the LCx emits various obtuse marginal branches to supply blood to the posterior part of the inferoposterior as well as posterolateral wall. As a result, the vector of injury current is directed more to the right and inferior in RCA occlusions, and more to the left and posterior in LCx occlusions $(14,15)$. We strongly believe that this minor difference in vector direction forms the basis of ECG differentiation between RCA and LCx occlusions.

In the present analysis of patients with acute IWMI, we observed that the $71 \%$ had a culprit lesion in the RCA and $29 \%$ with culprit lesion in the LCx vessel. This suggests that the RCA is significantly more likely to contain the culprit lesion compared with the LCx in patients with acute IWMI (ratio 2.4). Other studies involving patients with acute IWMI have reported the culprit vessel ratio of RCA to LCX in range from 2.2 to $7.0(16-18)$.

\section{Limitations}

The present study did not predict the precise location of lesions, either in the proximal or distal segment of a coronary vessel, or mega artery involvement. Furthermore, only patients with a first occurrence of STEMI were analyzed. The present study lacked follow-up data regarding complications such as reinfarction, postinfarction angina, arrhythmias and mortality. It should also be noted that the majority of enrolled patients experienced onset of chest pain within $6 \mathrm{~h}$ before ECG recording; therefore, the ECG findings in our study may differ considerably from patients with late presentation $(>12 \mathrm{~h})$ or with previous episodes of MI. Therefore, findings of the present study cannot be generalized to all patients with inferior-wall STEMI. We strongly emphasize that ECG-based algorithms should not replace coronary angiography. However, using these ECG criteria may lead to earlier identification of the culprit artery.

\section{CONCLUSION}

The existing ECG algorithms have good sensitivity for RCA and good specificity for LCx localization, while the specificity for RCA and sensitivity for LCx ranges from poor to fair. ST depression $\geq 0.5 \mathrm{~mm}$ in lead aVR as a predictor of LCx localization had a sensitivity of $90 \%$ and negative predictive value of $94.1 \%$. Furthermore, using ST depression $\geq 2 \mathrm{~mm}$ in lead aVR had a specificity of $91.4 \%$ for LCx localization. Thus, with inclusion of a single lead, we found both good sensitivity and specificity for LCx and RCA occlusions. Another parameter (ie, ST depression in aVR > aVL) also showed high specificity (97.1\%) for LCx localization. Although ECG-based algorithms should not be regarded as a replacement for coronary angiography, we believe that using these ECG criteria may lead to earlier identification of the culprit artery.

DISCLOSURES: The authors have no financial disclosures or conflicts of interest to declare. 


\section{REFERENCES}

1. Cassar A, Holmes DR Jr, Rihal CS, Gersh BJ. Chronic coronary artery disease: Diagnosis and management. Mayo Clin Proc 2009;84:1130-46.

2. Zimetbaum PJ, Josephson ME. Use of the electrocardiogram in acute myocardial infarction. N Engl J Med 2003;348:933-40.

3. Hosseini K, Bozorgi A, Karbalayi S. Predicting the culprit lesion in acute inferior ST-elevation myocardial infarction based on Wellens' criteria and Tierala's algorithm. Thrita 2014;3:e15607.

4. Sahi R, Sun J, Shah RK, Gupta M, Majagaiya BS. Clinical implication of ST segment depression in aVR \& aVL in patients with acute inferior wall myocardial infarction. World J Cardiovasc Dis 2015;5:278.

5. Kukla P, Bryniarski L, Dudek D, Krolikowski T, Kawecka Jaszcz K. Prognostic significance of ST segment changes in lead aVR in patients with acute inferior myocardial infarction with ST segment elevation. Kardiologia Polska 2012;70:111-8.

6. Fiol M, Cygankiewicz I, Carrillo A, et al. Value of electrocardiographic algorithm based on "ups and downs" of ST in assessment of a culprit artery in evolving inferior wall acute myocardial infarction. Am J Cardiol 2004;94:709-14.

7. Kosuge M, Kimura K, Ishikawa T, et al. New electrocardiographic criteria for predicting the site of coronary artery occlusion in inferior wall acute myocardial infarction. Am J Cardiol 1998;82:1318-22.

8. Tierala I, Nikus KC, Sclarovsky S, Syvanne M, Eskola M. Predicting the culprit artery in acute ST-elevation myocardial infarction and introducing a new algorithm to predict infarctrelated artery in inferior ST-elevation myocardial infarction: Correlation with coronary anatomy in the HAAMU trial. J Electrocardiol 2009;42:120-7.

9. Almansori M, Armstrong P, Fu Y, Kaul P. Electrocardiographic identification of the culprit coronary artery in inferior wall ST elevation myocardial infarction. Can J Cardiol 2010;26:293-6.
10. Elhammady W. aVR, the neglected electrocardiographic lead: Diagnostic and prognostic value in inferior ST-elevation myocardial infarction. HMJ 2013;7:71-6.

11. Kanei Y, Sharma J, Diwan R, et al. ST-segment depression in aVR as a predictor of culprit artery and infarct size in acute inferior wall ST-segment elevation myocardial infarction. J Electrocardiol 2010;43:132-5.

12. Nair R, Glancy DL. ECG discrimination between right and left circumflex coronary arterial occlusion in patients with acute inferior myocardial infarction: Value of old criteria and use of lead aVR. Chest 2002;122:134-9.

13. Sun TW, Wang LX, Zhang YZ. The value of ECG lead aVR in the differential diagnosis of acute inferior wall myocardial infarction. Intern Med (Tokyo, Japan) 2007;46:795-9.

14. Birnbaum Y, Drew BJ. The electrocardiogram in ST elevation acute myocardial infarction: Correlation with coronary anatomy and prognosis. Postgrad Med J 2003; 79:490-504.

15. Jim MH, Tsui KL, Yiu KH, et al. Jeopardised inferior myocardium (JIM) score: An arithmetic electrocardiographic score to predict the infarct-related artery in inferior myocardial infarction. Ann Acad Med - Singapore 2012;41:300.

16. Birnbaum Y, Wagner GS, Barbash GI, et al. Correlation of angiographic findings and right (V1 to V3) versus left (V4 to V6) precordial ST-segment depression in inferior wall acute myocardial infarction. Am J Cardiol 1999;83:143-8.

17. Gupta A, Lokhandwala Y, Kerkar P, Vora A. Electrocardiographic differentiation between right coronary and left circumflex coronary arterial occlusion in isolated inferior wall myocardial infarction. Ind Heart J 1998;51:281-4.

18. Zimetbaum PJ, Krishnan S, Gold A, Carrozza JP II, Josephson ME. Usefulness of ST-segment elevation in lead III exceeding that of lead II for identifying the location of the totally occluded coronary artery in inferior wall myocardial infarction. Am J Cardiol 1998;81:918-9. 